\title{
Behavioural plasticity in the fanning response of bumblebee workers: impact of experience and rate of temperature change
}

\author{
C. Westhus ${ }^{\text {a,b }}$, C. J. Kleineidam ${ }^{\text {a,c }}$, F. Roces ${ }^{a}$, A. Weidenmüller ${ }^{\text {a,c,* }}$ \\ ${ }^{a}$ Lehrstuhl für Verhaltensphysiologie und Soziobiologie, Biozentrum, Universität Würzburg, Würzburg, Germany \\ ${ }^{\mathrm{b}}$ Ecologie et Evolution, UPMC, Paris, France \\ ${ }^{\mathrm{c}}$ Fachbereich Biologie, Universität Konstanz, Konstanz, Germany
}

Keywords:

behavioural plasticity

Bombus terrestris

bumblebee

division of labour

experience

response threshold model

self-organization

self-reinforcement

thermoregulation
Flexible division of labour is a key feature of social insects. Our understanding of individual response behaviour and how it is integrated into a functioning colony is still patchy. Most theoretical studies addressing this question are based on the assumption that workers have intrinsic and often fixed response thresholds for task related stimuli. Here, we investigated the plasticity in worker fanning response behaviour in a bumblebee, Bombus terrestris. Using a temperature controlled brood dummy, we first asked whether the fanning response is modulated by rate of temperature change. Second, we examined to what extent the fanning response is influenced by recent fanning experience. Our results show that the individual fanning response is modulated by both the rate of temperature increase and recent experience. Workers responded at lower temperatures and with a higher probability when the temperature of the brood dummy increased slowly compared with more rapid temperature increases. Workers that repeatedly responded to an increase in brood dummy temperature with fanning showed a significant decrease in their response thresholds, whereas the response thresholds of control workers that experienced the same treatment but did not gather fanning experience remained unchanged. The decrease in response threshold was pronounced when the time interval between two successive fanning events was less than $6 \mathrm{~h}$. When $16 \mathrm{~h}$ or more separated fanning events, individual fanning responses returned to higher threshold levels. We suggest that experience dependent modulation of response thresholds plays an important role in the behavioural differentiation of workers and the flexibility of insect colonies.
Division of labour is a key property of insect societies. Workers perform different subsets of all tasks necessary for colony func tioning and are allocated to those tasks without any central control. It is commonly assumed that division of labour among the workers of a colony is the primary reason for the unsurpassed ecological success of social insects (Oster \& Wilson 1978; Hölldobler \& Wilson 1990).

One of the most remarkable features of division of labour is that colonies display an amazing degree of plasticity and resiliency. They respond to external changes and internal demands by adjusting the ratios of workers allocated to the various tasks; and they are able to compensate quickly for the removal of a substantial proportion of their workforce by rapid reallocation of workers from other tasks (Theraulaz et al. 1991; Robinson 1992; Gordon 1996). Our understanding of the mechanisms integrating individual

\footnotetext{
* Correspondence: A. Weidenmüller, Fachbereich Biologie, Universität Konstanz, 78457 Konstanz, Germany.

E-mail address: Anja.Weidenmueller@uni-konstanz.de (A. Weidenmüller).
}

behaviour into these amazingly flexible yet robust colony systems is still limited. What are the mechanisms underlying division of labour, and how is colony flexibility implemented at the level of the individual worker?

The current paradigm for division of labour is the response threshold model. It is built on the simple assumption that workers have intrinsic response thresholds for task associated stimuli, and that differences in response thresholds between the workers of a colony give rise to division of labour (reviewed in Beshers \& Fewell 2001). The majority of models assume individual response thresholds to be fixed. However, individual flexibility through experience and learning is presumably one of the key mechanisms underlying short term plasticity of colony responses.

Reinforcement models incorporate experienced based plasticity in response behaviour: performance of a task induces a decrease in the corresponding response threshold of a worker, whereas the lack of opportunity or failure in performance has the opposite effect (Plowright \& Plowright 1988; Theraulaz et al. 1998). Other models assume that individual response thresholds are modulated through social interactions (O'Donnell \& Bulova 2007). 
Response threshold models have been explored extensively from a theoretical perspective and have provided a much better understanding of the mechanisms underlying colony organization. However, models necessarily make simplifying assumptions con cerning behavioural decision rules. The behaviour of workers within a natural colony is bound to be much more complex. In response to encountering a stimulus repeatedly and/or performing the corresponding task repeatedly, individual behaviour may be modified in different ways.

First, almost all behavioural responses are fine tuned through learning, and experience may modulate task performance itself, i.e. the speed, efficiency, location or precision of a behaviour. Most empirical studies on the effect of experience in social insects have focused on such changes in task performance: for example, in foraging, nest repair, comb building, orientation (reviewed in Chittka \& Muller 2009) or recruitment (Franklin et al. 2012).

Second, experience may modulate individual response behav iour towards the stimulus. As assumed in the reinforced threshold models, experienced workers may respond to a stimulus at lower or higher stimulus intensities (change their response threshold). They may also respond with a lower or higher probability (change their response probability). In contrast to response threshold models, individual response threshold and response probability may be two independent parameters (Weidenmüller 2004) and changes in both parameters result in changes of individual responsiveness.

It is important to note that experience dependent changes in task performance and in task responsiveness may be two inde pendent processes. In ants, task performance may change with increasing task experience, while the responsiveness towards the task associated stimulus may remain unchanged (Weidenmüller et al. 2009).

Empirical evidence for a direct link between an individual's experience and its responsiveness towards a stimulus is scarce (Weidenmüller 2004; Ravary et al. 2007; Robinson et al. 2012).

Individual response behaviour may be modulated not only through recent experience, but also through characteristics of the stimulus itself. Although some task related stimuli may signify the necessity of a task simply by being present (e.g. comb damage and resulting nest repair, or alarm pheromone triggering nest defence), others, such as temperature, are continuously present and their variation may contain information that modulates individual responses to them. In the context of the control of nest climate, for instance, individuals may display thermoregulatory behaviours as a direct response to absolute temperatures, or to the rate of thermal change (Yousif 2005). Thus, in contrast to the assumption made in most theoretical models, individual response thresholds may not be fixed to one stimulus intensity. Rather, they may result from modulation through several parameters, one of which could be the rate of stimulus change.

Thermoregulation is an ideal system to study both individual and collective plasticity in response behaviour, as temperature naturally undergoes both daily and seasonal changes and can be easily measured and manipulated, and the corresponding behav ioural responses can be observed unambiguously. Like most social insects, bumblebees are able to control their nest temperature. This provides considerable independence from environmental condi tions and ensures rapid and safe development of the brood. The two thermoregulatory measures employed are incubation (to increase temperature) and wing fanning (to increase evaporative cooling and thereby cooling). These measures allow colonies to maintain their brood at $28-32{ }^{\circ} \mathrm{C}$, even under strongly fluctuating environ mental conditions (Schultze Motel 1991; Weidenmüller et al. 2002). Workers in a colony differ in their fanning response thresholds (O'Donnell \& Foster 2001; Weidenmüller 2004; Gardner et al. 2007). Moreover, a study on Bombus terrestris provided evidence for experience dependent changes in responsiveness: repeated exposure of colonies to temperature stress led to a decrease in individual temperature response thresholds of the fanning workers (Weidenmüller 2004). These findings support the reinforcement models; but they are in contrast to recent findings in B. impatiens, in which no effect of experience on individual fanning response thresholds was observed (Duong \& Dornhaus 2012). Thus, at the moment there is conflicting evidence for experience based modulation of response thresholds in bumblebee workers. Furthermore, to date no empirical study has provided evidence for the proposed 'negative reinforcement' by not performing a task that is assumed in the reinforced threshold model by Theraulaz et al. (1998).

In order to understand how the behaviour of numerous indi viduals is integrated into a functioning colony, we need a detailed knowledge of individual response decisions and of how individual response behaviour is modulated. In this study, we investigated the plasticity in the fanning response behaviour of $B$. terrestris workers on a temperature controlled brood dummy. First, we exposed workers to different temperature ramps and addressed the ques tion of whether experiencing a fast versus a more gradual change in brood temperature modulates individual response thresholds associated with fanning behaviour. Second, we repeatedly exposed workers to a fixed increase in brood temperature and asked whether recent experience with performing the task of fanning modulates a worker's responsiveness towards a temperature change.

\section{METHODS}

\section{Laboratory Colonies}

Young Bombus terrestris (L.) bumblebee colonies were obtained from a commercial breeder (Koppert B.V., The Netherlands) and consisted of the queen and up to 26 workers at delivery. They were housed in wooden boxes $(16 \times 28 \mathrm{~cm}$ and $10 \mathrm{~cm}$ high $)$ with Plexiglas covers inside a climate chamber $\left(21.8 \pm 1.4{ }^{\circ} \mathrm{C}, 12: 12 \mathrm{~h}\right.$ light:dark cycle). The boxes consisted of a nest chamber and a food chamber and had four screened ventilation holes. Colonies were supplied with ad libitum sucrose solution; defrosted, fresh honeybee packed pollen was given directly into the nest chambers. Newly emerged workers (identified through their pale coloration, which lasts for approximately $24 \mathrm{~h}$ ) were individually marked with numbered plastic tags (Opalithplättchen). Over the course of the experiments, colony sizes increased up to 140 workers; colonies were no longer used for experiments once they started producing sexuals.

To ensure that tested workers were naïve to the task of temperature fanning, colonies were kept at $22^{\circ} \mathrm{C}$; no insulation material was supplied and colonies were prevented from covering their nests by regularly removing canopy wax. Colonies were checked twice a day for fanning workers; these were observed only rarely (presumably $\mathrm{CO}_{2}$ fanners); their identities were noted and they were not used for experiments. Experiments were performed between October 2008 and July 2009 at the University of Würz burg, Germany.

\section{Experimental Set up}

The set up consisted of eight circular test arenas (diameter: $7.3 \mathrm{~cm}$, height: $4.9 \mathrm{~cm}$ ) made of Plexiglas (Fig. 1a). Each test arena had 12 ventilation holes, two indentations where sugar solution could be provided and a central temperature controlled aluminium pin that served as a brood dummy. The base of each aluminium pin (diameter $1 \mathrm{~cm}$ ) connected to a water filled aluminium heating 
(a)

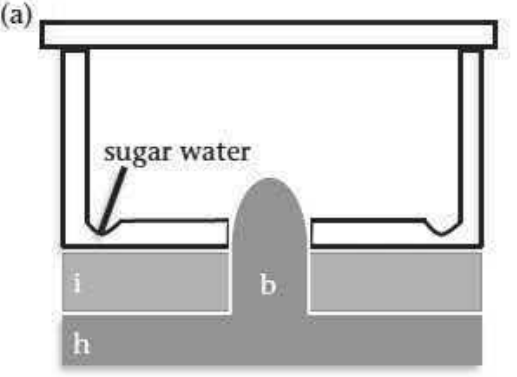

(b)

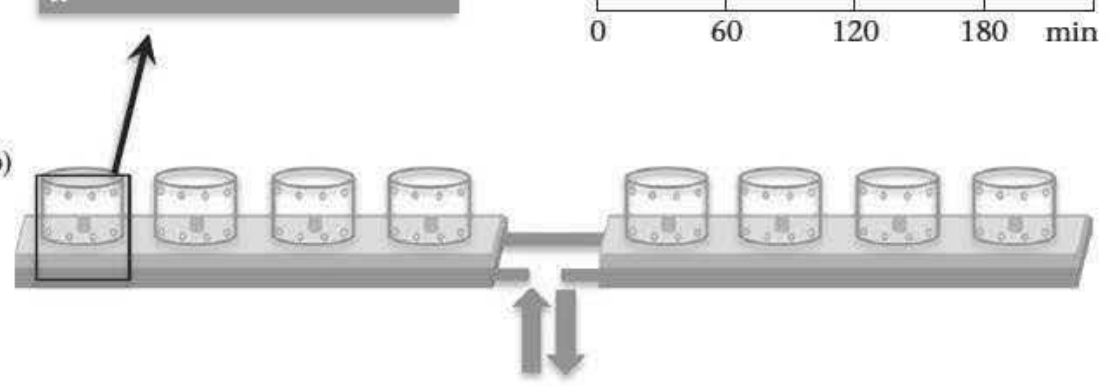

(c)

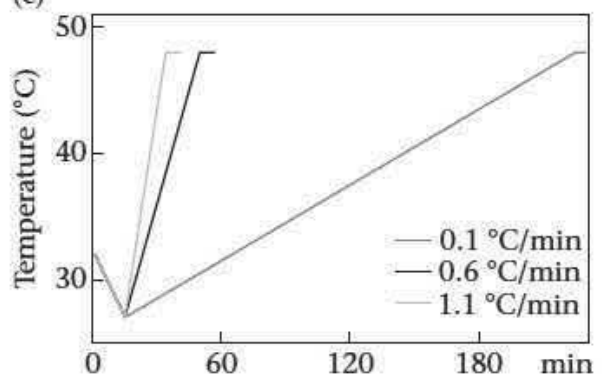

Figure 1. (a) Individual bumblebees were exposed to a temperature-controlled brood dummy (b). The dummy consisted of an aluminum pin that reached from a heating plate (h) through an insulating layer (i) into a test arena. The pin was covered with brood wax; sugar water was offered in small indentations on the side. (b) Test arenas had ventilation holes. The behaviour of eight bees was recorded in parallel. Brood dummy temperature was varied via a connected water bath. (c) In independent experiments, brood dummy temperature increased at $0.1,0.6$ or $1.1^{\circ} \mathrm{C} / \mathrm{min}$.

plate; the rounded tip extended through a styrofoam layer $(2.5 \mathrm{~cm})$ and reached $3.5 \mathrm{~mm}$ into the test arenas. The heating plate was connected to a programmable water bath (F25 ME JULABO USA), allowing controlled manipulation of brood dummy temperature.

In order to function as a brood dummy, the aluminium pin was covered with a thin layer of wax. For this purpose, wax was collected regularly from all colonies. Using wooden sticks, small amounts of canopy wax and of wax covering larvae were removed from various parts of the nest, placed separately between Parafilm layers and stored at $20^{\circ} \mathrm{C}$ until use.

\section{Experimental Procedure}

Experiments were conducted in the same climate chamber that housed the colonies to ensure stable environmental conditions and short transportation times of individual bees to the experimental set up. At the start of each experiment, brood dummies were prepared by covering each aluminium pin with $70-80 \mathrm{mg}$ of canopy wax, followed by $8 \mathrm{mg}$ of defrosted larvae wax. Brood dummy temperature was set to $32^{\circ} \mathrm{C}$, reflecting optimum brood tempera ture (Weidenmüller et al. 2002). Workers were randomly chosen from the brood area of their colony. With forceps, they were carefully transferred to the test arenas where they were left undisturbed for $20 \mathrm{~min}$ before tests began. Sucrose solution was offered ad libitum. In all experiments, workers were tested alone (one worker per test arena). Eight bees were tested in parallel (Fig. 1b).

During testing, the temperature of the brood dummies was experimentally modified following a fixed temperature regime. During the first $15 \mathrm{~min}$, it dropped from 32 to $27^{\circ} \mathrm{C}$ in all experi ments. Such an initial drop in temperature increases the propensity to perform thermoregulative brood care behaviour on the brood dummy (A. Weidenmüller, personal observation). Brood dummy temperature then increased at a constant rate (at $0.1,0.6$ or $1.1^{\circ} \mathrm{C}$ / min, depending on the experiment) to a maximum temperature of $48.3 \pm 0.5^{\circ} \mathrm{C}$, where it stayed constant for $8 \mathrm{~min}$ (Fig. 1c). Each bee remained in her test arena until the end of a trial.

During an experiment, the bees in the test arenas were contin uously observed. Fanning behaviour was recorded together with the time of its occurrence. 'Fanning' was defined as fanning with spread wings while standing still for at least $5 \mathrm{~s}$. Preliminary tests showed that wing fanning up to $4 \mathrm{~s}$ cannot clearly be distinguished from short wing fluttering, which occurs when bees are first faced with increasing temperatures. It is also displayed in antagonistic inter actions (Duchateau 1989) or during foraging (Dornhaus and Chittka, 2001), and can therefore not clearly be defined as thermoregulative behaviour. At the end of an experiment, workers were weighed (digital scale; Model AS60S, OHAUS) and returned to their colonies. Test arenas were cleaned and new wax was applied to the aluminium pins before the next experiment started. Experiments were performed between 1030 and 1800 hours.

\section{Effect of Rate of Temperature Increase}

To investigate whether fanning as a thermoregulatory response is triggered at an absolute temperature or depends on the rate of thermal increase, workers in test arenas were exposed to a temperature increase of $1.1,0.6$, or $0.1^{\circ} \mathrm{C} / \mathrm{min}$ (beginning at $27^{\circ} \mathrm{C}$ ). We tested 214 workers from nine different colonies $(21 \pm 18$ workers per colony; range 6-45). Forty six individuals were tested on the $0.1^{\circ} \mathrm{C} / \mathrm{min}$ temperature ramp, 114 individuals on the $0.6^{\circ} \mathrm{C} /$ min temperature ramp and 54 individuals on the $1.1^{\circ} \mathrm{C} / \mathrm{min}$ temperature ramp. Experiments were performed during different times of day. Workers of all ages were used for the experiments, except bees younger than 2 days.

\section{Effect of Recent Experience with the Task}

In order to measure the influence of recent fanning experience on individual fanning response behaviour, 131 workers from 11 different colonies were tested ( $12 \pm 6$ workers per colony; range 8-22). Workers were 4-8 days old when experiments started. During the experiments, they were repeatedly removed from their colonies to the test arenas. Workers were divided into two groups.

In the experimental group ( $\left.\begin{array}{ll}N & 48\end{array}\right)$, workers were exposed to a temperature increase of $0.6^{\circ} \mathrm{C} /$ min during nine subsequent trials. Three trials were performed per day: the first in the morning 
(1030-1145 hours); the second in the early afternoon (1315-1545 hours); and the third in the late afternoon (15451730 hours). In between subsequent trials, the bees were placed back into their colonies for 40100 min or overnight.

In the control group ( $N$ 83), workers received the same treatment but were exposed to an increase in brood temperature only during the first and the last trial. In the seven trials in between, brood dummy temperature was kept constant at $32{ }^{\circ} \mathrm{C}$, which does not elicit fanning behaviour.

\section{Data Analysis}

For analysis, the time of occurrence of fanning during an experiment could be assigned to the corresponding wax tempera ture of the brood dummy (to the nearest $0.1^{\circ} \mathrm{C}$ ). This was possible because before the experiments, for each brood dummy the change in its wax temperature during an experimental trial had been recorded three times using thermocouples (Thermologger K204 Conrad Electronic), and verified using an infrared thermometer (testo $860 \mathrm{~T} 1$ ). Wax temperature fluctuated no more than $\pm 0.3{ }^{\circ} \mathrm{C}$ between measurements.

Individual fanning response thresholds were defined as the lowest temperature at which fanning behaviour occurred. Workers that showed fanning before brood dummy temperature increased could not be assigned a temperature response threshold and were not included in the analysis. Fanning probability was measured as the proportion of individuals that fanned for a given condition.

The effect of rate of temperature increase on fanning response thresholds was analysed using a linear mixed model (LMM) with the fixed effects rate of temperature increase, bumblebee body mass and time of day, and the random effect colony. The full model included all these effects and a log transformed response variable to achieve normal error distribution. Each fixed factor was tested by removing the factor of interest from the previously chosen model; only significant fixed factors were kept to obtain a minimal adequate model. Models with and without the factor of interest were compared using a likelihood ratio test. A Tukey HSD test was applied as post hoc test on the means. The effect of rate of temperature increase on fanning probability was analysed using a general linear mixed model (GLMM) with the fixed effects rate of temperature increase, bumblebee mass and time of day, and the random effect colony. A Pearson's chi square test with a Yates correction for multiple testing was applied as post hoc test on the fanning probabilities of each temperature ramp.

To analyse the effect of experience on individual response thresholds, only workers that responded at least six times and during both the first and the last trial were included in the analysis. The change in response threshold between the first and last trial was calculated by subtracting the fanning temperature of the first trial from the fanning temperature in the last trial for each indi vidual. A $t$ test was applied due to normally distributed residuals to test whether the change in response threshold of both treatment groups was significantly different from zero, and to compare the change in response threshold between the two groups. To analyse an effect of experience on individual response probability, a McNemar chi square test for paired binomial distributed data was applied.

To investigate whether the time that had elapsed since the last responded trial (a trial during which a worker had responded to the temperature increase of the brood dummy with fanning) affected the change in response threshold, data of workers from the experimental group were further analysed. For all workers that had fanned in at least three of the nine trials, the change in response threshold between successive responded trials was calculated, and data were grouped into two categories: (1) change in thresholds over two successive responded trials that occurred within up to $6 \mathrm{~h}$ (within 1 day); and (2) change in thresholds over two successive responded trials that were separated by at least one night ( $>16 \mathrm{~h}$ ). As we were interested in a possible 'reset' of thresholds, or 'forgetting after learning', we looked at data from workers that had gathered fanning experience in at least two trials within 1 day prior to experiencing a delay in fanning of $16 \mathrm{~h}$ or longer.

The effect of time elapsed between two fanning events on the change in response thresholds was analysed using an LMM with the random effects colony and individual to control for repetitive measures of the same individual. Models with and without the factor time delay were compared using a likelihood ratio test. Integrated $t$ tests analysed whether the mean change in thresholds of the two time categories were significantly different from zero.

Statistical analyses were performed using the $\mathrm{R}$ software ( $\mathrm{v}$. 2.11.1, http://www.r project.org/); only two tailed tests were per formed. Descriptive statistics are presented as mean \pm SD, if not specified otherwise. All $P$ values above 0.05 were considered not statistically significant.

\section{RESULTS}

\section{Behaviour of Workers in the Experimental Set up}

After being transferred to the test arenas, workers were usually calm; escape attempts were hardly ever observed. Workers showed interest in the brood dummy by antennating or walking around it. Eighty per cent of all tested bees ( $N$ 345; from 11 colonies) showed thermoregulatory behaviour towards the brood dummy (incubating and/or fanning); 48\% showed fanning behaviour when brood dummy temperature increased. Most of the workers fanned on or near the brood dummy; some had contact with the brood dummy and subsequently fanned somewhere else in the test arena. Once a worker had started fanning, she would do so intermittently, sometimes walking around, checking the wax with her antennae or drinking sugar water between fanning events. Uninterrupted fanning usually lasted for up to $1 \mathrm{~min}$; the longest uninterrupted fanning we observed lasted $332 \mathrm{~s}$. Air temperature within the test arena (measured $1.5 \mathrm{~cm}$ from the brood dummy) never exceeded $25^{\circ} \mathrm{C}$; workers responded exclusively to the change in wax temperature of the dummy.

\section{Response Behaviour and Rate of Temperature Increase}

The rate of stimulus increase influenced both fanning response probability and fanning response thresholds of the tested workers. Of all bees entering this experiment $\left(\begin{array}{ll}N & 214\end{array}\right), 78 \%$ showed fanning in response to an increase in brood dummy temperature when it increased at $0.1^{\circ} \mathrm{C} / \mathrm{min}$, compared with $52 \%$ when temperature increased at $0.6^{\circ} \mathrm{C} / \mathrm{min}$ and only $33 \%$ when temperature increased at $1.1^{\circ} \mathrm{C} / \mathrm{min}$ (Fig. 2a). Thus, a faster temperature increase led to a significantly lower response probability (GLMM: $\chi_{2}^{2}$ 23.590, $P<0.001$ ). Further post hoc tests supported the significant differ ence of response probabilities between each temperature ramp (Pearson chi square test: 0.1 versus $0.6^{\circ} \mathrm{C} / \mathrm{min}: \chi_{1}^{2} \quad 8.007$, Yates corrected $P \quad 0.005 ; 0.6$ versus $1.1^{\circ} \mathrm{C} / \mathrm{min}: \chi_{1}^{2} \quad 4.294$, Yates cor rected $P \quad 0.038 ; 0.1$ versus $1.1^{\circ} \mathrm{C} / \mathrm{min}: \chi_{1}^{2} \quad 16.551$, Yates cor rected $P<0.001$ ). Fanning probability was not affected by time of day (GLMM: $\chi_{1}^{2}$ 2.597, $P$ 0.107), but it was affected by bumblebee body mass (GLMM: $\chi_{1}^{2} \quad 6.407, P \quad 0.011$ ). Tested bees weighed $110-550 \mathrm{mg}$ (mean + SD $281+70 \mathrm{mg}, N \quad 106)$ and had a higher probability of fanning when they were smaller (regression coefficient in the GLMM 5.347, SE 2.144).

Response thresholds were also modulated by the rate of temperature increase (Fig. 2b; LMM: L ratio 2 21.006, $P<0.001$ ). 

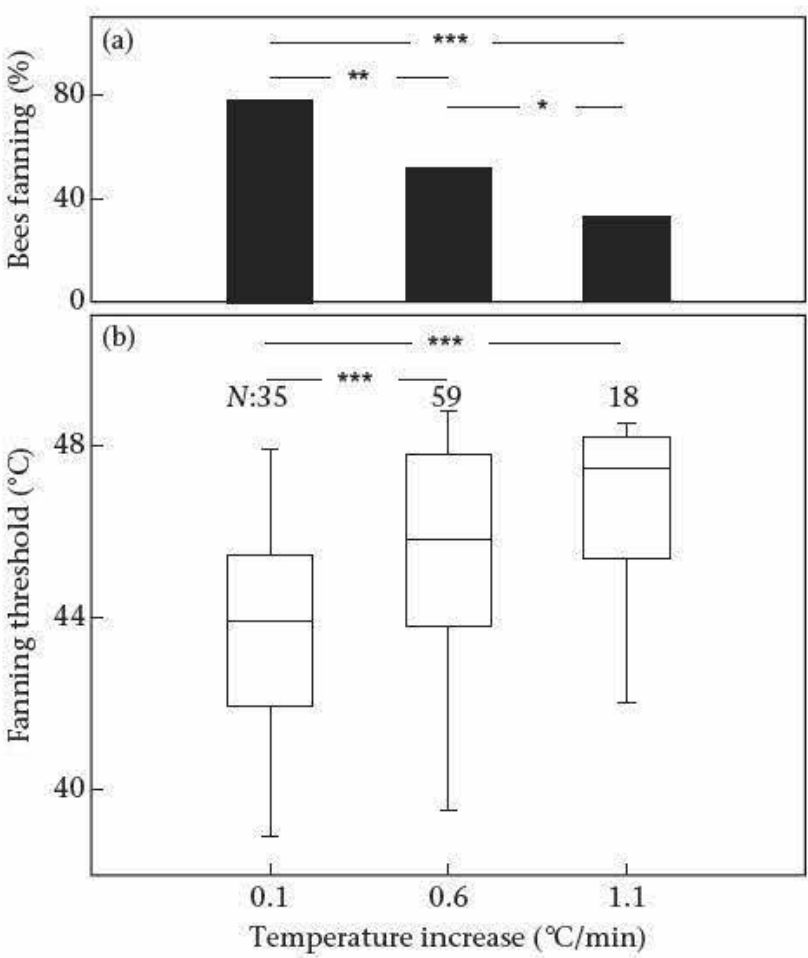

Figure 2. (a) The percentage of tested bees fanning and (b) their fanning response thresholds in relation to the rate of temperature increase of the brood dummy $\left({ }^{* * *} P<0.001 ;{ }^{* *} P<0.01 ;{ }^{*} P<0.05\right)$. Box plots show median, quartile and range. $N$ denotes numbers of responding individuals.

Whereas workers fanned at $43.3 \pm 3.2^{\circ} \mathrm{C}$ when temperature increased at $0.1^{\circ} \mathrm{C} / \mathrm{min}$, they first fanned at $45.6 \pm 1.8^{\circ} \mathrm{C}$ when temperature increased at $0.6^{\circ} \mathrm{C} / \mathrm{min}$, and at $46.6 \pm 2.1^{\circ} \mathrm{C}$ when temperature increased at $1.1^{\circ} \mathrm{C} / \mathrm{min}$. This corresponds to $170 \pm 20 \mathrm{~min}, 48 \pm 4 \mathrm{~min}$ and $32 \pm 2 \mathrm{~min}$ after the start of the experiment for the $0.1,0.6$ and $1.1^{\circ} \mathrm{C} / \mathrm{min}$ ramp, respectively. Colony origin had no significant effect on response thresholds (Akaike information criterion (AIC) values of: (1) full mod el 239.214; (2) model without random factor 241.214). A post hoc comparison test on the means revealed that response thresholds were significantly higher at temperature increases of 0.6 and $1.1^{\circ} \mathrm{C} / \mathrm{min}$ compared with $0.1^{\circ} \mathrm{C} / \mathrm{min}$ (Tukey HSD test: both adjusted $P<0.001$ ). However, response thresholds did not signifi cantly differ between a temperature increase of $0.6^{\circ} \mathrm{C} / \mathrm{min}$ and one of $1.1^{\circ} \mathrm{C} / \mathrm{min}$ (Tukey HSD test: adjusted $P \quad 0.239$ ). There was no effect of body mass (LMM: $L$ ratio $1 \quad 2.045, P \quad 0.153$ ) or time of day (LMM: L ratio 7 6.506, $P$ 0.482) on individual response thresholds. Worker weight did not differ significantly between colonies (linear model: $F_{8,105} \quad 1.683, P \quad 0.112$ ).

\section{Response Behaviour and Recent Experience with the Task}

The influence of recent experience on individual fanning response behaviour to increasing temperatures was investigated in 131 workers. In the control group, 83 workers were exposed to increasing temperatures during their first and last (ninth) trial only, experiencing seven trials without temperature increase in between. Three bees showed spontaneous fanning behaviour at $32{ }^{\circ} \mathrm{C}$ and were not included in the analysis. Only 12 bees (14\%) fanned during both the first and the last trial and could be used for comparison with the experimental group.

In the experimental group, 48 workers were exposed to increasing temperatures in each of the nine consecutive trials. Thirteen (27\%) of these bees were unresponsive (i.e. they never fanned), 11 (23\%) fanned in fewer than six of the nine trials, 24 (50\%) fanned six times or more. Of these 'frequent fanners', 17 fanned during both the first and the last trial and could be used for a comparison with the control group.

To compare the two groups, we analysed the change in fanning response threshold between the first and last trial for each indi vidual. Response thresholds of workers in the control group did not change between first and last trial ( $t$ test: $t_{11} \quad 0.033, P \quad 0.974$; Fig. 3). In the frequent fanners from the experimental group that fanned during both the first and the last trial, fanning response thresholds were significantly lower when measured during the last trial compared with the first trial, decreasing by about $4{ }^{\circ} \mathrm{C}$ $\left(\begin{array}{lll}t_{16} & 4.905, P & 0.0002\end{array}\right)$. The change in thresholds of these frequent fanners was significantly different from the control group $\left(\begin{array}{lll}t_{27} & 3.124, P & 0.004\end{array}\right)$. Previous experience with fanning behav iour in response to increasing brood temperature therefore modulated individual fanning response thresholds.

Response probability of workers in the experimental group was around $50 \%$ and not affected by experience. Fifty eight per cent ( $N$ 28) of all workers fanned during the first trial; $48 \%$ fanned during the last trial (McNemar chi square test: $\chi_{1}^{2} 1.455$, $\begin{array}{ll}P & 0.228) \text {. }\end{array}$

Despite the overall decrease in the fanning response thresholds from first to last trial found in the frequent fanners, the decrease was not linear across the nine trials. This may be partly because not every worker responded in each trial, and the time interval between successive fanning events differed. Workers that gained fanning experience and responded several times over the course of 1 day tended to show a decrease in fanning threshold, but they often exhibited an increased response threshold during the first test of the following day, especially after the first night. Figure 4 illustrates the response thresholds of 21 workers from the experi mental group that responded during the first trial and then at least five more times over following trials, compared with the responses of 12 workers from the control group.

\section{Response Behaviour and Time Elapsed since last Task Performance}

To address the question whether the time span between two successive responded trials impacts the change in response

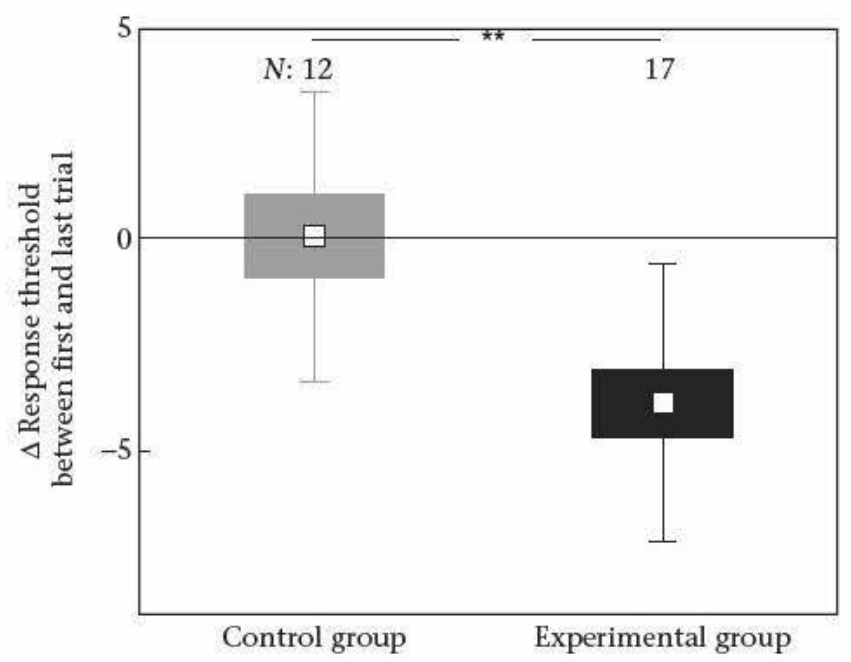

Figure 3. Difference in fanning response thresholds between the first and the last (ninth) trial, compared for workers from the control and the experimental group $\left({ }^{* *} P<0.01\right)$. Workers in the experimental group gathered experience in fanning as response to a repeated increase in brood temperature, whereas control workers were exposed to a brood temperature increase only in the first and the last trial. Box plots show mean \pm SE and \pm SD. 


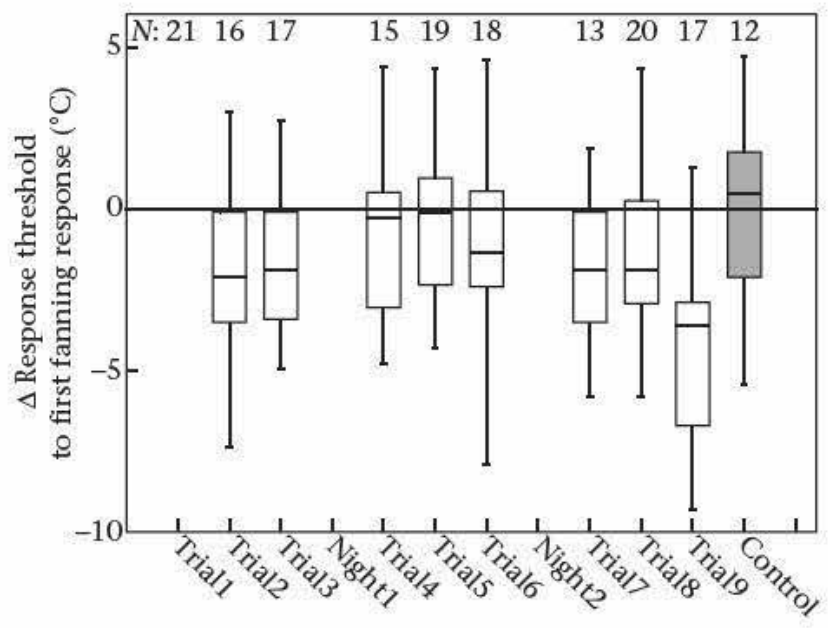

Figure 4. Change in individual fanning response thresholds of experienced workers over the nine experimental trials. For each individual and each trial, the difference in response threshold to the individuals' response threshold during the first trial is shown. Response thresholds of 21 workers that fanned during the first trial and then at least five more times are shown. For comparison, fanning response thresholds of 12 workers from the control group during their ninth trial are shown in the grey box. These workers experienced an increase in temperature only during the first and the last (ninth) trial. Box plots show median, quartile and range without outliers.

threshold, we further analysed the data of 32 workers that responded at least three times in the experimental group. The change in individual response threshold between successive responded trials depended on the time interval between the two responded trials (LMM: $L 1$ 12.457, $P<0.001$ ). When the two responded trials were separated by no more than $6 \mathrm{~h}$, response thresholds decreased significantly by $1.0+0.3^{\circ} \mathrm{C}(\chi+\mathrm{SE}, N \quad 116$; $t$ test: $\left.t_{134}-3.267, P \quad 0.001\right)$. By contrast, when the two responded trials were separated by $16 \mathrm{~h}$ or more, and workers had previously gathered some experience (i.e. responded to an increase in brood dummy temperature at least twice before experiencing such a long delay) thresholds increased significantly by $1.0+0.5^{\circ} \mathrm{C}$ (mean + SE, $N \quad 51 ; t$ test: $\left.t_{134} \quad 2.122, P \quad 0.036\right)$.

\section{DISCUSSION}

The main objective of this study was to investigate the plasticity in the response behaviour of thermoregulating bumblebees. We analysed the modulatory effect of two different parameters on fanning response behaviour: the rate of stimulus increase experi enced by the workers, and experience with performing the task. Our results document that individual response thresholds in bumblebees are not fixed. Workers do not merely respond by fanning once an absolute temperature threshold is exceeded; they integrate temporal information into their fanning response deci sion. Furthermore, fanning response thresholds are modulated by previous experience in performing the task of fanning.

We used a new experimental approach in studying the ther moregulatory response behavilowr of bumblebees. Workers were individually tested on a wax covered brood dummy, so that potential social feedbacks arising from nestmates were completely controlled for. During experimerits, only the temperature of the brood dummy was manipulated whereas air temperature in the test arenas and body temperature of the wrorkers remained unmanipulated. The fact that workers responded to an increase in brood dummy temperature (and resumably some olfactory cues present in the brood wax) by fanming demonstrates that they assess and control the temperature of the brood itself. In previous studies on individual fanning response behaviour, nest temperature was increased using heating lamps (Weidenmüller 2004; Gardner et al. 2007; Duong \& Dornhaus 2012). This approach has the drawback that a constant stimulus increase is very hard to achieve, and that stimulus intensities may differ strongly depending on where in a colony (i.e. how exposed to the thermal radiation from the lamp) a worker is during the experiment. Exposure to heat radiation has a strong impact on the body temperature of the dark bees, and nest air temperature, which is often used as measure for stimulus intensity and for determining individual response thresholds, may not be indicative of actual stimulus intensity perceived by the workers.

Under natural conditions, body temperature of workers in the nest and brood temperature are closely linked, and both are presumably relevant for a response at adequate temperatures. This may explain why fanning response thresholds measured in the present study are higher than the optimal brood temperature of around $32{ }^{\circ} \mathrm{C}$ (Weidenmüller et al. 2002) and the fanning response thresholds measured for workers fanning in heat stressed colonies (O'Donnell \& Foster 2001; Weidenmüller 2004; Duong \& Dornhaus 2012). Despite this 'shift' in response thresholds, the probability of fanning in workers exposed to an increase in brood dummy temperature was similar to the response probability reported for workers exposed to an increase in air temperature to $30-35^{\circ} \mathrm{C}$ within colonies (O'Donnell \& Foster 2001; Weidenmüller 2004).

\section{Rate of Temperature Increase modulates Response Behaviour}

Nest temperature in many social insects undergoes fluctuations (Jones \& Oldroyd 2006). These fluctuations can be rapid or slow; nest temperature can increase as a result of changes in ambient air temperature and solar radiation, and the metabolic heat produced by colony members. Do workers integrate information about the rate of stimulus change into their decision to respond to a temper ature increase? Testing the fanning response of individual workers on different temperature ramps revealed that fanning is not elicited when an absolute temperature threshold is exceeded; rather, the rate of stimulus increase impacts the fanning response decision of the workers.

Contrary to intuitive prediction, workers responded at signifi cantly lower temperatures and with a significantly higher proba bility when brood dummy temperature increased slowly compared with when it increased more rapidly. Workers showed the highest temperature tolerance when temperature increased at a rate of $1.1^{\circ} \mathrm{C} / \mathrm{min}$. Similar findings have been reported for temperature responses of brood tending ants (Yousif 2005).

Bombus terrestris nests are found mainly in underground cavi ties, where a colony may be more likely to experience gradual temperature fluctuations, which it can dampen by responding quickly and with a large number of workers. It is possible that the sensory system of bumblebees is adapted to follow the low rate of temperature change but is less suited to cope with the highest rates of temperature change we used in our experiments.

One possible mechanistic explanation for the differences in response thresholds is that any delay between the perception of dangerously high brood temperatures and the execution of the corresponding behaviour (fanning) would shift the threshold values to higher levels on the steep temperature inclines. Further more, workers that experienced a temperature increase of $0.1^{\circ} \mathrm{C}$ spent over $2.5 \mathrm{~h}$ on the gradually warming brood dummy before their fanning response thresholds were exceeded. In comparison, workers that experienced an increase of $1.1^{\circ} \mathrm{C}$ experienced less than $20 \mathrm{~min}$ of temperature increase before their fanning thresh olds were exceeded. In order to uncouple the possible effect of time elapsed, execution delay and temperature, further experiments 
comparing the response behaviour of individuals to constant temperature increases with that of temperature increases following step functions are needed.

Workers in bumblebee colonies vary considerably in size, and size is known to affect division of labour (e.g. Free 1955; Goulson et al. 2002; Spaethe \& Weidenmuller 2002; Jandt \& Dornhaus 2009). For the task of fanning, we found no correlation between response thresholds and body size, confirming findings of other studies (Weidenmüller 2004; Duong \& Dornhaus 2012). However, we found that smaller workers had a higher probability of fanning in response to an increase in brood dummy temperature. In B. huntii smaller bees have also been shown to incubate at higher rates (Gardner et al. 2007). In contrast to these findings, a study on $B$. impatiens reported that larger workers were more likely to fan than smaller workers (Jandt \& Dornhaus 2009). This inconsistency may result from species specific differences or differences in the experimental design: in the study by Jandt \& Dornhaus, workers were observed in unmanipulated colonies that were not exposed to an experimental temperature increase. It is therefore possible that these fanning workers were responding to $\mathrm{CO}_{2}$ rather than to an increase in nest temperature.

In conclusion, our results show that exposure to a change in temperature over time has an impact on the response behaviour of bumblebee workers, demonstrating that response thresholds are not fixed to one absolute temperature.

\section{Experience in Task Performance Modulates Response Thresholds}

Experience affects all aspects of animal behaviour. In social insects, the behaviour of numerous individuals is integrated into one functioning unit, the colony. Surprisingly, our understanding of the impact of experience on the behavioural differentiation of workers within colonies is still limited. The results of the present study show that the repeated performance of fanning behaviour under controlled laboratory conditions results in a decrease in fanning response threshold of bumblebee workers; after nine trials, workers that had frequently fanned responded to the same temperature increase at significantly lower temperatures. This effect was not found in the control group that experienced the same experimental treatment except for the temperature increase during trials $2-8$. We therefore propose that the observed decrease in response thresholds of the experienced workers was not due to stress, ageing or any other factor besides experience.

The finding of experience dependent changes in response thresholds are in accordance with an earlier study on fanning behaviour in $B$. terrestris, in which workers that repeatedly expe rienced a temperature increase within their colonies showed a decrease in fanning response thresholds (Weidenmüller 2004). Duong \& Dornhaus (2012), however, found no effect of experience on response thresholds in Bombus impatiens; in this study workers gained fanning experience when exposed to heat lamp induced temperature stress in groups outside their colonies. The differ ence between the results of these studies may be due to species specific differences, or the difficulties of controlled stimulus application discussed above.

In the present study, a decrease in response thresholds was evident even after only one trial; response thresholds were usually already markedly lower during the second trial. A strong effect of the first experience in task performance has also been reported for ants (Langridge et al. 2004, 2008), although whether individual behavioural thresholds to a specific stimulus decreased in this case remains elusive.

The finding of decreasing thresholds as a consequence of repeatedly performing a task supports assumptions underlying the reinforcement models (Theraulaz et al. 1998). In these models, a worker's response threshold decreases when the corresponding task is performed. Importantly, the models assume that thresholds are flexible in both directions; when a task is not performed, thresholds increase.

Empirical support for a decrease in responsiveness based on nonperformance of the corresponding task is extremely scarce. In the context of task performance, a reduction in, for example, nectar intake rate, pollen collection rate and size of pollen loads in bumblebee foragers after the first night following the first learning trials has been demonstrated (e.g. Keasar et al. 1996; Raine \& Chittka 2007). A study on colony emigration in Temnothorax albi pennis ants showed that colonies behave as if they have no previous experience of emigration when the time span between emigrations is above 6 days (Langridge et al. 2004).

Our results provide empirical evidence for changes in respon siveness through nonperformance: when $6 \mathrm{~h}$ or less had passed between two successive fanning events, the fanning thresholds of experienced workers decreased. However, if experienced workers had not fanned for $16 \mathrm{~h}$ or more, we found a significant increase, or 'reset' in their thresholds. This effect was especially prominent after the first night. These findings also support the idea that it is indeed task performance itself, and not experiencing the increase in temperature, that modulates responsiveness.

The mechanisms underlying the time dependent change in response thresholds remain unclear. Experience induced changes in behaviour imply some form of learning. How workers learn and what the possible 'reinforcers' are in the context of thermoregu lation remains to be investigated. Do workers assess their own efficiency in performing a task, and does efficiency impact future responses (Ravary et al. 2007; Robinson et al. 2012)? Is the reset of thresholds following longer delays between fanning events due to partial retention; and would it not occur if more fanning trials preceded a delay?

Workers in our experiments were clearly limited in their ability to cool the surface temperature of the brood dummy beneath them efficiently. In previous experiments we often observed that workers fanned for longer durations and with a higher probability when brood dummy temperature beneath them was experimentally decreased (not increasing or stable, as in the current study) while they were fanning. In future studies, we will address the potential effect of individual efficiency on response behaviour and learning by directly manipulating individual fanning efficiency.

Experience dependent and reversible modulation of response thresholds may be an important mechanism underlying colony flexibility. When a colony frequently experiences a high demand for a task (e.g. when overheating of the nest occurs on a daily basis) an increasing number of workers gather experience with the task of fanning. As a consequence, the response thresholds of these workers will decrease and they will start responding to a temper ature increase at lower temperatures. At the colony level this will presumably result in smaller nest temperature fluctuations. When high demand for a task ceases, for example when the weather changes and the nest no longer experiences regular overheating, the thresholds of those workers will shift back up; they will be less 'sensitive' to a temperature change and 'freed' for other, potentially more urgent tasks.

It is important to note that while response thresholds were clearly modulated by experience in performing the corresponding task, we found no change in response probability with increasing experience. Previous studies have shown that response threshold and response probability are two independent parameters of fanning response behaviour in bumblebees (Weidenmüller 2004). In honeybees, patrilines have been shown to differ more strongly in their response probability for recruitment signalling than in their corresponding response thresholds (Mattila \& Seeley 2010). 
Interindividual differences in both parameters of worker respon siveness, response thresholds and response probability, may play an important role in the behavioural differentiation of workers and in the flexibility and robustness of division of labour (Weidenmüller 2004; Duong \& Dornhaus 2012). In the present study, nearly half the workers did not respond to an increase in temperature; and of the workers that did respond, some fanned almost every time temperature increased, and others only very rarely even though their response thresholds were exceeded. Workers with the lowest response thresholds and the highest response probabilities will be the most likely to gain experience in fanning and thus undergo a decrease in their response thresholds. Their fanning behaviour will lower the stimulus level in the colony and thereby decrease the probability that higher threshold workers also engage in this task. Thus, small intrinsic variations between workers may be amplified through experience into larger differ ences in task performance (Page \& Mitchell 1998).

\section{Conclusions}

Understanding the complexity of an insect society requires a sound knowledge of the rules that govern the individual decision making of its members. Our results show that the fanning response behaviour of bumblebee workers is not fixed to one stimulus intensity. Rather, responsiveness is modulated both by how a change in temperature is experienced and by repeated experience with the performance of the task itself. We suggest that in addition to the effects of experience on task performance, experience dependent modulation in response thresholds, as demonstrated in the present study, plays a decisive role for the behavioural differentiation of workers and the flexibility of insect colonies.

\section{Acknowledgments}

We thank S. Pielström for fruitful discussions, S. Pielström and C. Doums for help with data analysis, A. Gerber Kurz for assistance with colony maintenance and D. Missoh for his support. We thank three anonymous referees and C. Doums for constructive comments on the manuscript. We are grateful to the German Research Foundation (DFG, grant SFB 554) for funding.

\section{References}

Beshers, S. N. \& Fewell, J. H. 2001. Models of division of labor in social insects Annual Review of Entomology, 46, 413440.

Chittka, L. \& Muller, H. 2009. Learning, specialization, efficiency and task allocation in social insects. Communicative \& Integrative Biology, 2, 151154

Dornhaus, A. \& Chittka, L. 2001. Food alert in bumblebees (Bombus terrestris): possible mechanisms and evolutionary implications. Behavioral Ecology and Sociobiology, 50, 570576

Duchateau, M. 1989. Agonistic behaviours in colonies of the bumblebee Bombus terrestris. Journal of Ethology, 7, 141151.

Duong, N. \& Dornhaus, A. 2012. Ventilation response thresholds do not change with age or self-reinforcement in workers of the bumble bee Bombus impatiens. Insectes Sociaux, 59, 2532.

Franklin, E. F., Robinson, E. J. H., MarshallJ, A. R., Sendova-FranksA, B. \& Franks, N. R. 2012. Do ants need to be old and experienced to teach? Journal of Experimental Biology, 215, 12871292.
Free, J. B. 1955. The division of labour within bumblebee colonies. Insectes Sociaux, 11, 195212.

Gardner, K. E., Foster, R. L. \& O'Donnell, S. 2007. Experimental analysis of worker division of labor in bumblebee nest thermoregulation (Bombus huntii, Hymenoptera: Apidae). Behavioral Ecology and Sociobiology, 61, 783792.

Gordon, D. M. 1996. The organization of work in social insect colonies. Nature, $\mathbf{3 8 0}$ 121124.

Goulson, D., Peat, J., Stout, J. C., Tucker, J., Darvill, B., Derwent, L. C. \& Hughes, W. O. 2002. Can alloethism in workers of the bumblebee, Bombus terrestris, be explained in terms of foraging efficiency? Animal Behaviour $64,123130$.

Hölldobler, B. \& Wilson, E. O. 1990. The Ants. Cambridge, Massachusetts: Harvard University Press.

Jandt, J. M. \& Dornhaus, A. 2009. Spatial organization and division of labour in the bumblebee. Bombus impatiens. Animal Behaviour, 77, 641651.

Jones, J. C. \& Oldroyd, B. P. 2006. Nest thermoregulation in social insects. Advances in Insect Physiology, 33, 153191.

Keasar, T., Motro, U., Shur, Y. \& Shmida, A. 1996. Overnight memory retention of foraging skills by bumblebees is imperfect. Animal Behaviour, 52, 95104

Langridge, E. A., Franks, N. R. \& Sendova-Franks, A. B. 2004. Improvement in collective performance with experience in ants. Behavioral Ecology and Sociobiology, 56, 523529.

Langridge, E. A., Sendova-Franks, A. B. \& Franks, N. R. 2008. How experienced individuals contribute to an improvement in collective performance in ants. Behavioral Ecology and Sociobiology, 62, 447456.

Mattila, H. R. \& Seeley, T. D. 2010. Promiscuous honeybee queens generate colonies with a critical minority of waggle-dancing foragers. Behavioral Ecology and Sociobiology, 64, 875889.

O'Donnell, S. \& Bulova, S. J. 2007. Worker connectivity: a simulation model of variation in worker communication and its effects on task performance. Insectes Sociaux, 54, 211218.

O'Donnell, S. \& Foster, R. L. 2001. Thresholds of response in nest thermoregulation by worker bumble bees, Bombus bifarius nearcticus (Hymenoptera: Apidae). Ethology, 107, 387399.

Oster, G. F. \& Wilson, E. O. 1978. Caste and Ecology in the Social Insects. Princeton, New Jersey: Princeton University Press.

Page, R. E. \& Mitchell, S. D. 1998. Self-organization and the evolution of division of labor. Apidologie, 29, 171190.

Plowright, R. C. \& Plowright, C. M. S. 1988. Elitism in social insects: a positive feedback model. In: Interindividual Behavioral Variability in Social Insects (Ed. by R. L. Jeanne), pp. 419 432. Boulder, Colorado: Westview Press.

Raine, N. E. \& Chittka, L. 2007. Pollen foraging: learning a complex motor skill by bumblebees (Bombus terrestris). Die Naturwissenschaften, 94, 459464.

Ravary, F., Lecoutey, E., Kaminski, G., Châline, N. \& Jaisson, P. 2007. Individual experience alone can generate lasting division of labor in ants. Current Biology, 17, 13081312.

Robinson, E. J. H., Feinerman, O. \& Franks, N. R. 2012. Experience, corpulence and decision making in ant foraging. Journal of Experimental Biology, 215 26532659.

Robinson, G. E. 1992. Regulation of division of labor in insect societies. Annual Review of Entomology, 37, 637665.

Schultze-Motel, P. 1991. Heat loss and thermoregulation in a nest of the bumblebee Bombus lapidarius (hymenoptera, apidae). Thermochimica Acta, 193, 5766

Spaethe, J. \& Weidenmuller, A. 2002. Size variation and foraging rate in bumblebees (Bombus terrestris). Insectes Sociaux, 49, 142146.

Theraulaz, G., Gervet, J. \& Tian-Chanski, S. S. 1991. Social regulation of foraging activities in Polistes dominulus Christ: a systemic approach to behavioural organization. Behaviour, 116, 292320.

Theraulaz, G., Bonabeau, E. \& Deneubourg, J. L. 1998. Response threshold reinforcement and division of labour in insect societies. Proceedings of the Royal Society B, 265, 327332 .

Weidenmüller, A. 2004. The control of nest climate in bumblebee (Bombus terrestris) colonies: interindividual variability and self reinforcement in fanning response. Behavioral Ecology, 15, 120128.

Weidenmüller, A., Kleineidam, C. \& Tautz, J. 2002. Collective control of nest climate parameters in bumblebee colonies. Animal Behaviour, 63, 10651071.

Weidenmüller, A., Mayr, C., Kleineidam, C. J. \& Roces, F. 2009. Preimaginal and adult experience modulates the thermal response behavior of ants. Current Biology, 19, 18971902

Yousif, J. 2005. Die Thermoperiodizität und die Kontrolle der Bruttemperatur bei der Ameisenart Camponotus mus. Diploma thesis, Fakultät für Biologie, JuliusMaximilians-Universität, Würzburg. 